\title{
UNE INÉGALITÉ DE CHEEGER POUR LE SPECTRE DE STEKLOV
}

\author{
par
}

Pierre Jammes

\begin{abstract}
Résumé. - On montre une inégalité de Cheeger pour la première valeur propre de Steklov. Elle fait intervenir deux constantes isopérimétriques.
\end{abstract}

Abstract. - We prove a Cheeger inequality for the first positive Steklov eigenvalue. It involves two isoperimetric constants.

Soit $M$ une variété compacte à bord et $\gamma \in C^{1,1}(M), \rho \in C^{0}(\partial M)$ deux fonctions densités strictement positives sur $M$ et $\partial M$ respectivement. Le problème aux valeurs propres de Steklov consiste à résoudre l'équation, d'inconnues $\sigma \in \mathbb{R}$ et $f: \bar{M} \rightarrow \mathbb{R}$,

$$
\begin{cases}\operatorname{div}(\gamma \nabla f)=0 & \text { dans } M \\ \gamma \frac{\partial f}{\partial \nu}=\sigma \rho f & \text { sur } \partial M\end{cases}
$$

où $\nu$ est un vecteur unitaire sortant normal au bord. On parle de problème de Steklov homogène quand $\gamma \equiv 1$ et $\rho \equiv 1$, et le cas $\gamma \not \equiv 1$ se rattache au problème de Calderón. L'ensemble des réels $\sigma$ solutions du problème forme un spectre discret positif noté

$$
0=\sigma_{0}(M, g, \rho, \gamma)<\sigma_{1}(M, g, \rho, \gamma) \leq \sigma_{2}(M, g, \rho, \gamma) \ldots
$$

C'est le spectre d'un opérateur Dirichlet-Neumann $H^{1}(\partial M) \rightarrow L^{2}(\partial M)$ défini par $\Lambda_{\rho, \gamma} u=\frac{\gamma}{\rho} \frac{\partial \mathcal{H}_{\gamma} u}{\partial \nu}$, où $\mathcal{H}_{\gamma} u$ est le prolongement harmonique de $u$ pour la densité $\gamma$, c'està-dire que $\operatorname{div}\left(\gamma \nabla\left(\mathcal{H}_{\gamma} u\right)\right)=0$. Il est auto-adjoint pour la norme de Hilbert $\|u\|^{2}=$ $\int_{\partial M} u^{2} \rho \mathrm{d} v_{g}$ (voir [Ban80, [SU90] et [Uhl09]). Ce spectre possède une caractérisation variationnelle; on a en particulier pour la première valeur propre :

$$
\sigma_{1}(M, g, \rho, \gamma)=\inf _{\substack{f \in H^{1}(\bar{M}) \\ \int_{\partial M} f^{2} \rho \mathrm{d} v_{g}=0}} \frac{\int_{M}|\mathrm{~d} f|^{2} \gamma \mathrm{d} v_{g}}{\int_{\partial M} f^{2} \rho \mathrm{d} v_{g}} .
$$

L'objet de cet article est de donner une minoration de $\sigma_{1}(M)$ en fonction d'invariants isopérimétriques qui est analogue à l'inégalité de Cheeger pour la première valeur propre du laplacien. Pour ce faire, on introduit les notations suivantes : si $D$ est un domaine de $M$, on note $\partial_{E} D=\partial D \cap \partial M$ le bord « extérieur » de $D$ et $\partial_{I} D=\partial D \backslash \partial_{E} D$ son

Classification mathématique par sujets (2000). - 35P15, 58J50.

Mots clefs. — inégalité de Cheeger, spectre de Steklov. 
bord intérieur. Les volumes $n$ et $(n-1)$-dimensionnels seront calculés relativement aux densités $\gamma$ et $\rho$ et notés $|\cdot|_{\gamma}$ et $|\cdot|_{\rho}$. On pose alors

$$
h(M, g, \gamma)=\inf _{|D|_{\gamma} \leq \frac{|M|_{\gamma}}{2}} \frac{\left|\partial_{I} D\right|_{\gamma}}{|D|_{\gamma}} \text { et } h^{\prime}(M, g, \rho, \gamma)=\inf _{|D|_{\gamma} \leq \frac{|M|_{\gamma}}{2}} \frac{\left|\partial_{I} D\right|_{\gamma}}{\left|\partial_{E} D\right|_{\rho}} .
$$

Quand $\gamma \equiv 1$, la constante $h$ est la constante de Cheeger classique; la première valeur propre non nulle du laplacien de Neumann sur $M$ est minorée par $h^{2} / 4$ ([Che70, Bus80]). Des analogues dans le cas $\gamma \not \equiv 1$ ont été introduits dans [Bro85] et C097. La constante $h^{\prime}$ intervient spécifiquement dans le problème de Steklov. Après avoir écrit la première version de cet article, j'ai appris que J. Escobar définit une constante presque identique à $h^{\prime}$ dans [Esc97] dans le but de minorer $\sigma_{1}$. Mais l'inégalité qui suit, plus simple et plus explicite que celle de [Esc97], semble avoir échappé aux recherches menées jusqu'à présent :

Théorème 1. - Soit $(M, g)$ une variété riemannienne compacte à bord munie des densités $\rho$ et $\gamma$ sur $\partial M$ et $M$. On a $h^{\prime}(M)>0$ et $\sigma_{1}(M) \geq \frac{h(M) \cdot h^{\prime}(M)}{4}$.

Remarque 2. - On peut en fait montrer deux inégalités différentes : si on modifie légèrement les définitions de $h$ et $h^{\prime}$ en remplaçant la condition $|D|_{\gamma} \leq|M|_{\gamma} / 2$ par $\left|\partial_{E} D\right|_{\rho} \leq|\partial M|_{\rho} / 2$, la démonstration reste valide mais les constantes isopérimétriques ne sont plus les mêmes. En particulier, la constante $h^{\prime}$ est remplacée par la constante définie par Escobar.

Remarque 3. - Si on multiplie la métrique par $\lambda^{2}(\lambda>0)$, alors $\sigma_{1}$ et $h$ sont divisées par $\lambda$ et $h^{\prime}$ est invariant. Pour des raisons d'homogénéité, on ne peut donc pas avoir une minoration de $\sigma_{1}$ de la forme $c \cdot h^{\alpha} \cdot h^{\prime \beta}$ où $c>0, \alpha \neq 1$ et $\beta \in \mathbb{R}$ sont des constantes universelles. Le cas $\alpha=1$ et $\beta<1$ est exclu par l'exemple qui suit.

Exemple 4. - Soit $(M, g)$ une variété close. Le spectre de Steklov (homogène) de $M_{n}=M \times\left[0, \frac{1}{n}\right]$ a été calculé dans [CESG11] (lemme 6.1) et $\sigma_{1}\left(M_{n}\right) \sim \frac{1}{n}$ quand $n \rightarrow \infty$. On peut encadrer $h\left(M_{n}\right)$ uniformément : étant donné un domaine $D$ de $M_{n}$, on considère $2 n$ copies de $M_{n}$ qu'on recolle le long de leurs bords pour obtenir une variété $M \times S^{1}$ dont la métrique est indépendante de $n$, et on construit un domaine $D^{\prime} \subset M \times S^{1}$ en recollant les domaines $D$ par réflexion le long du bord. On obtient alors $\left|D^{\prime}\right|=2 n|D|$ et $\left|\partial D^{\prime}\right|=2 n\left|\partial_{I} D\right|$ donc $\left|\partial_{I} D\right| /|D|=\left|\partial D^{\prime}\right| /\left|D^{\prime}\right| \geq h\left(M \times S^{1}\right)$ et $h\left(M_{n}\right) \geq h\left(M \times S^{1}\right)$. En considérant un domaine de la forme $D=U \times\left[0, \frac{1}{n}\right]$ avec $U \subset M$, on voit que $h\left(M_{n}\right)$ est majoré par une constante.

On peut aussi estimer $h^{\prime}$ : l'exemple d'un domaine $U \times\left[0, \frac{1}{n}\right]$ montre que $h^{\prime}\left(M_{n}\right) \leq \frac{c}{n}$ pour une constante $c>0$. Réciproquement, pour un domaine $D$ fixé, $\left|\partial D_{E}\right|$ est indépendant de $n$ et le volume de $\partial D_{I}$ sur $M_{n}$ vérifie $\left|\partial D_{I}\right|_{n} \geq\left|\partial D_{I}\right|_{1} / n$. Par conséquent, $h^{\prime}\left(M_{n}\right) \geq h^{\prime}\left(M_{1}\right) / n$.

On voit que sur cet exemple, l'exposant de $h^{\prime}$ dans l'inégalité est optimal.

Exemple 5. - On considère encore la variété $M \times[0, L]$ où $M$ est une variété close, mais on suppose que $L \rightarrow+\infty$. Dans ce cas, on a $\sigma_{1}=1 / L$. Parmi les domaines $D$ de volume fixé suffisamment grand, l'aire de $\partial_{I} D$ est minimisée par les hypersurfaces $\partial_{I} D=M \times\{x\}$, par conséquent $h=2 / L$ et $h^{\prime}=1$. Dans cet exemple, c'est $h$ qui tend vers 0 quand $\sigma_{1} \rightarrow 0$ et pas $h^{\prime}$. 
Exemple 6. - Dans GP10 (section 2), A. Girouard et I. Polterovich construisent une famille de domaines du plan (formés de deux disques reliés par une anse fine) pour lesquels $\sigma_{1}, h$ et $h^{\prime}$ tendent vers zéro. Contrairement à la remarque et aux exemples qui précèdent, le volume reste borné inférieurement et supérieurement.

Exemple 7. - Les 《haltères de Cheeger généralisées » construites par P. Guérini dans Gué04 fournissent des exemples à mi-chemin entre les exemples 4 et 6; on va ici étudier le cas de la dimension 3. Étant donné un $\varepsilon>0$, dans $\mathbb{R}^{3}$ muni d'un repère $(O x y z)$ on considère le cercle unité $S^{1}$ et le disque unité $D^{2}$ du plan $(O x y)$, et on définit les domaines $V_{1}=\left\{p \in \mathbb{R}^{3}, d\left(p, S^{1}\right)<\frac{1}{4}\right\}$ et $\left.V_{2}=D^{2} \times\right]-\varepsilon, \varepsilon[$. La variété définie par $M_{\varepsilon}=V_{1} \cup V_{2}$ est homéomorphe à une boule et sa constante isopérimétrique $h^{\prime}$ tend vers 0 quand $\varepsilon \rightarrow 0$ (considérer un domaine $D \subset M_{\varepsilon}$ de la forme $\left.U \times\right]-\varepsilon, \varepsilon$ [ avec $U \subset(O x y)$ contenu dans le disque de centre $O$ et rayon 3/4). On a aussi $\sigma_{1}\left(M_{\varepsilon}\right) \rightarrow 0$ : il suffit de fixer une fonction test $(O x y) \rightarrow \mathbb{R}$ d'intégrale nulle et à support dans le disque de centre $O$ et rayon $3 / 4$ et de la relever à $\mathbb{R}^{3}$. Dans la formule (3), le numérateur sera proportionnel à $\varepsilon$ tandis que le numérateur reste constant. En construisant de la même manière un espace test de dimension arbitrairement grande, on montre qu'en fait toutes les valeurs propres tendent vers 0 . Ce phénomène d' « effondrement du spectre » avait déjà été observé par A. Girouard et I. Polterovich sur l'exemple précédent.

Démonstration du théorème. - On fixe une métrique $g$ et des densités $\rho$ et $\gamma$. Pour montrer que $h^{\prime}>0$ il suffit de traiter le cas homogène, le cas général s'y ramène car les densités sont continues, donc encadrées par des constantes strictement positives. On se donne un $\varepsilon \in] 0,1]$ dont la valeur précise sera fixée plus tard. Si $\left|\partial_{E} D\right| \leq(1-\varepsilon)|\partial M|$, où $D$ est un domaine intervenant dans la définition de $h^{\prime}$, J. Escobar montre dans Esc99 que le rapport $\left|\partial_{I} D\right| /\left|\partial_{E} D\right|$ est minoré par une constante $K(\varepsilon)>0$ (il ne traite que le cas $\varepsilon=\frac{1}{2}$ mais sa démonstration se généralise immédiatement). Si $\left|\partial_{E} D\right| \geq$ $(1-\varepsilon)|\partial M|$ on utilise le fait que le volume de $M \backslash D$ est minoré par $|M| / 2$ pour tout $D$, et donc qu'il existe une constante $C(g)>0$ telle que $|\partial(M \backslash D)|>C$. On a alors $\left|\partial_{I} D\right|=|\partial(M \backslash D)|-\left|\partial M \backslash \partial_{E} D\right| \geq C+\left|\partial_{E} D\right|-|\partial M| \geq C-\varepsilon|\partial M|$. En choisissant $\varepsilon$ suffisamment petit par rapport à $C$ et $|\partial M|$, on a donc $\left|\partial_{I} D\right| \geq \varepsilon|\partial M| \geq \varepsilon\left|\partial_{E} D\right|$ ce qui permet de conclure.

On montre maintenant la minoration de $\sigma_{1}$. Afin d'alléger les notations on n'explicitera pas les références aux densités, celles-ci étant généralement évidentes. Soit $f$ une fonction propre sur $M$ de la première valeur propre $\sigma_{1}(M)$. On choisit son signe de sorte que $M^{+}=f^{-1}\left(\left[0,+\infty[)\right.\right.$ vérifie $\left|M^{+}\right| \leq|M| / 2$ (ou $\left|\partial_{E} M^{+}\right| \leq|\partial M| / 2$ si on suit la remarque 21). La fonction $f$ restreinte à $M^{+}$est alors la première fonction propre du problème de Steklov sur $M^{+}$avec condition de Dirichlet sur $\partial_{I} M^{+}$, pour la valeur propre $\sigma_{1}$. Rappelons que problème de Steklov avec condition de Dirichlet sur une partie du bord de la variété est bien défini, que son spectre est strictement positif et qu'on a aussi la relation $\sigma_{1}=\int_{M^{+}}|\mathrm{d} f|^{2} / \int_{\partial M^{+}} f^{2}$ (cf. Agr05). Par conséquent on peut écrire, en utilisant l'inégalité de Cauchy-Schwarz :

$$
\begin{aligned}
\sigma_{1} & =\frac{\int_{M^{+}}|\mathrm{d} f|^{2}}{\int_{\partial M^{+}} f^{2}}=\frac{\left(\int_{M^{+}} f^{2}\right)\left(\int_{M^{+}}|\mathrm{d} f|^{2}\right)}{\left(\int_{M^{+}} f^{2}\right)\left(\int_{\partial M^{+}} f^{2}\right)} \geq \frac{\left(\int_{M^{+}} f|\mathrm{~d} f|\right)^{2}}{\left(\int_{M^{+}} f^{2}\right)\left(\int_{\partial M^{+}} f^{2}\right)} \\
& \geq \frac{1}{4} \frac{\left(\int_{M^{+}}\left|\mathrm{d}\left(f^{2}\right)\right|\right)^{2}}{\left(\int_{M^{+}} f^{2}\right)\left(\int_{\partial M^{+}} f^{2}\right)}=\frac{1}{4} \frac{\int_{M^{+}}\left|\mathrm{d}\left(f^{2}\right)\right|}{\int_{M^{+}} f^{2}} \cdot \frac{\int_{M^{+}}\left|\mathrm{d}\left(f^{2}\right)\right|}{\int_{\partial M^{+}} f^{2}} .
\end{aligned}
$$


En posant $D_{t}=f^{-1}([\sqrt{t},+\infty[)$, la formule de la co-aire $([\underline{\text { KP08 }}]$, ch. 5$)$ donne les trois relations

(2) $\int_{M^{+}}\left|\mathrm{d}\left(f^{2}\right)\right|=\int_{t \geq 0}\left|\partial_{I} D_{t}\right| \mathrm{d} t, \int_{M^{+}} f^{2}=\int_{t \geq 0}\left|D_{t}\right| \mathrm{d} t$ et $\int_{\partial M^{+}} f^{2}=\int_{t \geq 0}\left|\partial_{E} D_{t}\right| \mathrm{d} t$.

Comme $\left|D_{t}\right| \leq\left|M^{+}\right| \leq|M| / 2$ (ou $\left|\partial_{E} D_{t}\right| \leq\left|\partial_{E} M^{+}\right| \leq|\partial M| / 2$ ) pour tout $t \geq 0$, on en déduit que $\int_{M^{+}}\left|\mathrm{d}\left(f^{2}\right)\right| \geq h \int_{M^{+}} f^{2}$ et $\int_{M^{+}}\left|\mathrm{d}\left(f^{2}\right)\right| \geq h^{\prime} \int_{\partial M^{+}} f^{2}$, et donc que $\sigma_{1} \geq \frac{1}{4} h h^{\prime}$

\section{Références}

[Agr05] M. S. Agranovich - « On a mixed Poincaré-Steklov Type Spectral Problem in a Lipschitz Domain », Russ. J. Math. Phys. 13 (2005), no. 3, p. 239-244.

[Ban80] C. BANDLE - Isoperimetric inequalities and applications, Monographs and Studies in Mathematics, vol. 7, Pitman, 1980.

[Bro85] R. BRooks - « The bottom of the spectrum of a riemannian covering », J. Reine Angew. Math. 357 (1985), p. 101-114.

[Bus80] P. Busen - « On Cheeger inequality $\lambda_{1} \geq h^{2} / 4 »$, in Geometry of the Laplace operator, Proc. Sympos. Pure Math., XXXVI, Amer. Math. Soc., 1980, p. 29-77.

[CESG11] B. Colbois, A. El Soufi \& A. Girouard - « Isoperimetric control of the Steklov spectrum », J. Funct. Anal. 261 (2011), no. 5, p. 1384-1399.

[Che70] J. ChEEger - «A lower bound for the smallest eigenvalue of the Laplacian », in Problems in analysis (Papers dedicated to Salomon Bochner, 1969), Princeton Univ. Press, 1970.

[CO97] S.-Y. Cheng \& K. Oden - « Isoperimetric inequalities and the gap between the first and second eigenvalues of an Euclidean domain », J. Geom. Anal. 7 (1997), no. 2, p. 217-239.

[Esc97] J. F. EscobAR - «The geometry of the first non-zero Stekloff eigenvalue », J. Funct. Anal. 150 (1997), no. 2, p. 544-556.

[Esc99] _ « An isoperimetric Inequality and the first Steklov Eigenvalue », J. Funct. Anal. 165 (1999), no. 1, p. 101-116.

[GP10] A. Girouard \& I. Polterovich - « On the Hersch-Payne-Schiffer inequalities for Steklov eigenvalues », Functional Analysis and its Applications 44 (2010), no. 2, p. 106-117.

[Gué04] P. Guérini - «Prescription du spectre du laplacien de Hodge-de Rham », Ann. scient. Éc. norm. sup. (4) 37 (2004), no. 2, p. 270-303.

[KP08] S. T. Krantz \& H. R. PARKS - Geometric Integration Theory, Birkäuser, 2008.

[SU90] J. Sylvester \& G. Uhlmann - « The Dirichlet to Neumann map and applications », in Inverse problems in partial differential equations (Arcata, CA, 1989), SIAM, 1990, p. 101139.

[Uhl09] G. Uhlmann - «Electrical impedance tomography and Calderón's problem », Inverse Problems 25 (2009), no. 12, p. 123011, 39.

Pierre Jammes, Univ. Nice Sophia Antipolis, CNRS, LJAD, UMR 7351, 06100 Nice, France E-mail : pjammes@unice.fr 